\title{
Identificación de Estrategias de Aprendizaje de Estudiantes de Ingeniería de Alimentos en los Cursos Balance de Materia, Transporte de Fluidos y Operaciones Unitarias
}

\author{
Diofanor Acevedo(1), Marlene Durán ${ }^{(2)}$ y Armando Alvis ${ }^{(3)}$ \\ (1) Universidad de Cartagena, Facultad de Ingeniería, Programa de Ingeniería de Alimentos, Grupo de \\ Investigación NUSCA, Avenida el Consulado, Calle 30 No. 48-152. Cartagena, Bolívar-Colombia \\ (e-mail: diofanor3000@gmail.com) \\ (2) Universidad de Cartagena, Facultad de Medicina, Programa de Medicina, Sede Zaragocilla. Cartagena, \\ Bolívar-Colombia. \\ (3) Universidad de Córdoba, Programa de Ingeniería de Alimentos, Facultad de Ingenierías, 6 No 76-103, \\ Km 3, vía Cereté. Córdoba-Colombia.
}

Recibido Ene. 29, 2015; Aceptado Abr. 13, 2015; Versión final Jul. 9, 2015, Publicado Dic. 2015

\begin{abstract}
Resumen
Se identifican las estrategias de aprendizajes de estudiantes en las asignaturas de balance de materia, transporte de fluidos, y operaciones unitarias, del programa ingeniería de alimentos de la Universidad de Cartagena. La muestra de estudio consistió de 130 alumnos, 46\% hombres de edad promedio 20.3 años y 54\% mujeres de edad promedio 21.9 años. Se aplicó el cuestionario ACRA que evaluó las escalas de Adquisición, Codificación, Recuperación y Apoyo. Se realizó análisis de varianza y se obtuvo una correlación entre las escalas usando la prueba de Pearson, La preferencia general estuvo orientada hacia las escalas de codificación y apoyo, siendo las mujeres las que presentaron promedios más altos en dichas escalas, en las tres asignaturas. Entre hombres y mujeres hubo diferencias estadísticamente significativas en cada una de las escalas analizadas $(p<0.05)$ por lo que se establece que el género si influye en la elección de las estrategias de aprendizaje.
\end{abstract}

Palabras clave: estrategias de aprendizaje, ingeniería de alimentos, cuestionario ACRA, influencia de género

\section{Identification of Learning Strategies in Students of Food Engineering: Courses Balance Matter, Fluid Transport and Unit Operations}

\begin{abstract}
The aim of this work was to identify learning strategies of students in the subjects of material balance, fluid transport, and unit operations of the food engineering program at the University of Cartagena. The sample consisted of 130 students, $46 \%$ men of average age 20.3 years and $54 \%$ women of average age 21.9 years. ACRA questionnaire that includes the scales Acquisition, Encoding, Retrieval and Support was applied. Analysis of variance was performed and a correlation between the scales using Pearson's test. The general preference was oriented to the coding and support scales, being women the ones who had higher averages in those scales, for the three subjects. Between men and women there were statistically significant differences in each of the analyzed scales $(p<0.05)$, concluding that gender influences the choice of the learning strategies.
\end{abstract}

Keywords: learning strategies, food engineering, ACRA questionnaire, gender influence 


\section{INTRODUCCIÓN}

Cada día la educación se encuentra en constante cambio, buscando contribuir en la formación de personas con capacidades de manejar información, por ello es importante entender la forma en que se asimilan los conocimientos (López-Aguado, 2011). Medina et al., (2014) señalan que las estrategias de aprendizaje (EDA) son el conjunto de actividades, técnicas y medios que los estudiantes planifican y realizan de acuerdo con sus necesidades de formación académica.

Quispe et al., (2014) indican que las EDA son conductas o pensamientos que facilitan la asimilación de los conocimientos. Estas van desde las simples habilidades de estudio, como el subrayado de la idea principal, hasta los procesos de pensamiento complejo (Pool-Cibrian y Martínez-Guerrero, 2013). De acuerdo con Villardón-Gallego et al., (2013) las estrategias cognoscitivas son las operaciones y los procedimientos que el estudiante utiliza para adquirir, retener y recuperar diferentes tipos de conocimientos. Indica además que involucran capacidades representacionales (como la lectura, imaginería, habla, escritura y dibujo), selectivas (como la atención y la intención) y autodireccionales (como la autoprogramación y el automonitoreo), y se componen de dos partes: una tarea cognoscitiva orientadora, y una o más capacidades representacionales, selectivas o autodireccionales (Gargallo et al., 2012).

Camarero et al., (2000) (Citado por Pestana y Martínez, 2013 y Bahamón et al., 2013) definen las EDA como actividades propositivas en cuatro grandes fases del procesamiento de la información, representadas en las escalas del cuestionario ACRA, desarrollado y validado por Román y Gallego (1994) (Citado por Román y Gallegos, 2001). Este formato ha sido empleado en diversos estudios, y revela las habilidades que experimenta una persona a la hora de aprender. El ACRA es un instrumento d autoinforme, inspirado en los principios cognitivos de procesamiento de la información, permite evaluar de forma cuantitativa diversas estrategias cognitivas generales que utilizan los estudiantes en el aprendizaje que tiene lugar durante la actividad de estudio, en sus distintas fases (Bahamón et al., 2013). En general los indicadores de validez y fiabilidad de la prueba son aceptables (Medina et al., 2014; Quispe et al., 2014; Muelas y Navarro et al., 2015). La adquisición de la información, se refiere cuando el individuo toma por primera vez gran parte de la información por medio de procesos atencionales y receptores sensoriales, hasta llegar a un registro en la memoria de corto plazo; en esta etapa es fundamental el proceso cognoscitivo de la atención, pues ésta se encarga de seleccionar, transformar y transportar la información desde el ambiente al registro sensorial (Bahamón et al., 2013). La codificación hace mención a las habilidades encargadas de fijar la información obtenida a la memoria de largo plazo requiriendo una mejor organización y haciendo más fácil su manejo, estas facilitan la elaboración y organización de la información relacionándola y dándole significados para generar nuevos modelos mentales (Gargallo et al., 2012). Estas se complementan con las de recuperación, que son las tácticas que ayudan a recordar la información almacenada a través de procesos de búsqueda y auto-respuesta en la memoria; y las de apoyo de la información, las cuales ayudan a regular el aprendizaje y se refieren a las destrezas que refuerzan a las anteriores y que se encuentran influenciadas por factores emocionales, motivacionales y sociales (Loret de Mola, 2011; Muelas y Navarro, 2015).

Marugán, et al., (2013) afirma que las EDA cognitivas varían según lo que se quiera aprender, ya que cada persona tiende a desarrollar ciertas preferencias de acuerdo a los métodos que le resulten más apropiados. Alkhateeb y Nasser, (2014) por su parte, afirman que si no se tiene certeza de la forma en que los alumnos aprenden, es muy difícil la creación métodos de enseñanza por parte de los educadores. Por tal razón, son muchos los problemas a los que se enfrentan los docentes a la hora de impartir el conocimiento, debido a que en la mayoría de los casos no conocen las estrategias que más utilizan los alumnos. En general identificar las EDA en alumnos, es importante, ya que puede ser una alternativa para mejorar las metodología de enseñanza-aprendizaje en los distintos centro educativos (Gallo et al., 2014). Por ello el objetivo de este trabajo fue identificar las principales estrategias de aprendizajes utilizadas por los estudiantes en las asignaturas de balance de materia, transporte de fluidos y operaciones unitarias, del programa Ingeniería de Alimentos de la Universidad de Cartagena.

\section{MATERIALES Y MÉTODOS}

Se realizó un estudio descriptivo, correlacional y de corte transversal, sobre variables no experimentales, durante los semestres 2013-2, 2014-1, 2014-2 y 2015-1, en el programa de Ingeniería de Alimentos de la Universidad de Cartagena (Colombia). Similar a lo realizado por Cassé et al., (2010), Quispe et al., (2014) y Muelas y Navarro, (2015).

\section{Recopilación de datos para analizar el las calificaciones de los estudiantes}

Las tres asignaturas fueron dictadas por el mismo docente durante el periodo de estudio analizado. Según el plan curricular del programa Ingeniería de Alimentos, balance de materia fue prerrequisito de transporte 
de fluidos y ésta última prerrequisito de operaciones unitarias. Las metodologías de enseñanza fueron similares, con prácticas de laboratorios, clases teóricas, discusiones grupales sobre temas de actualidad y seminarios con artículos de investigación proporcionados por las bases de datos científicas a las cuales se tiene acceso en la Universidad de Cartagena. La identificación del género de todos los estudiantes que cursaron las asignaturas y las calificaciones generales fueron recopiladas de las planillas de registro y control académico, suministradas por el docente del área. Se consideró una calificación mínima de 3,0 para la aprobación, en una escala de 0,0 a 5,0.

\section{Población y muestra}

La población objeto de estudio fueron 324 estudiantes, matriculados durante el periodo de estudio en las asignaturas correspondientes de balances de materia (26\%), transporte de fluido (35\%) y operaciones unitarias (40\%) correspondiente al ciclo de ciencias de la ingeniería del programa ingeniería de Alimentos de la Universidad de Cartagena. Para calcular el tamaño de la muestra representativa, se utilizó el procedimiento por estrato (López, 2004) que consistió en dividir a la población en subgrupos homogéneos, estableciendo proporciones, hasta obtener 130 unidades muéstrales, de las cuales el $46 \%$ fueron hombres con una edad promedio de $20,32 \pm 0,76$ años y el $54 \%$ mujeres con una edad de $21,91 \pm 0,85$. Distribuidos aleatoriamente en las respectivas asignaturas como se esquematiza en la Tabla 1.

Tabla 1: Distribución de la población y muestra objeto de estudio

\begin{tabular}{|c|c|c|c|c|c|c|c|c|c|c|}
\hline \multicolumn{11}{|c|}{ Tamaño Población } \\
\hline \multirow{2}{*}{$\begin{array}{c}\text { Asignaturas/Género } \\
\mathrm{s}\end{array}$} & \multicolumn{2}{|c|}{ 2013-II } & \multicolumn{2}{|c|}{ I } & \multicolumn{2}{|c|}{ 2014-II } & \multicolumn{2}{|c|}{ 2015-I } & \multicolumn{2}{|c|}{ Total } \\
\hline & $\begin{array}{c}\text { Hombre } \\
s\end{array}$ & $\begin{array}{l}\text { Mujere } \\
\mathrm{s}\end{array}$ & $\begin{array}{c}\text { Hombre } \\
\text { s }\end{array}$ & $\begin{array}{l}\text { Mujere } \\
\mathrm{S}\end{array}$ & $\begin{array}{c}\text { Hombre } \\
s\end{array}$ & $\begin{array}{l}\text { Mujere } \\
\mathrm{s}\end{array}$ & $\begin{array}{c}\text { Hombre } \\
s\end{array}$ & $\begin{array}{l}\text { Mujere } \\
\mathrm{s}\end{array}$ & $\begin{array}{c}\text { Hombre } \\
\text { s }\end{array}$ & $\begin{array}{l}\text { Mujere } \\
\mathrm{s}\end{array}$ \\
\hline Balance Materia & 13 & 15 & 12 & 10 & 6 & 12 & 8 & 8 & 39 & 45 \\
\hline Transporte Fluidos & 14 & 16 & 14 & 16 & 12 & 18 & 10 & 12 & 50 & 62 \\
\hline $\begin{array}{l}\text { Operaciones } \\
\text { Unitarias }\end{array}$ & 16 & 14 & 16 & 20 & 16 & 12 & 14 & 20 & 62 & 66 \\
\hline Total & 43 & 45 & 42 & 46 & 34 & 42 & 32 & 40 & 151 & 173 \\
\hline Total por semestre & \multicolumn{2}{|c|}{88} & \multicolumn{2}{|c|}{88} & \multicolumn{2}{|c|}{76} & \multicolumn{2}{|c|}{72} & \multicolumn{2}{|c|}{324} \\
\hline \multicolumn{11}{|c|}{ Tamaño Muestra } \\
\hline \multirow{2}{*}{$\begin{array}{c}\text { Asignaturas/Género } \\
\mathrm{s}\end{array}$} & \multicolumn{2}{|c|}{ 2013-II } & \multicolumn{2}{|c|}{ 2014 } & \multicolumn{2}{|c|}{ 2014-II } & \multicolumn{2}{|c|}{ 2015-I } & \multicolumn{2}{|c|}{ Total } \\
\hline & $\begin{array}{c}\text { Hombre } \\
s\end{array}$ & $\begin{array}{c}\text { Mujere } \\
\mathrm{S}\end{array}$ & $\begin{array}{c}\text { Hombre } \\
s\end{array}$ & $\begin{array}{c}\text { Mujere } \\
\mathrm{s}\end{array}$ & $\begin{array}{c}\text { Hombre } \\
s\end{array}$ & $\begin{array}{c}\text { Mujere } \\
\mathrm{s}\end{array}$ & $\begin{array}{c}\text { Hombre } \\
s\end{array}$ & $\begin{array}{c}\text { Mujere } \\
\mathrm{s}\end{array}$ & $\begin{array}{c}\text { Hombre } \\
s\end{array}$ & $\begin{array}{c}\text { Mujere } \\
\mathrm{s}\end{array}$ \\
\hline Balance Materia & 5 & 6 & 5 & 4 & 2 & 5 & 3 & 3 & 16 & 18 \\
\hline Transporte Fluidos & 6 & 6 & 6 & 6 & 5 & 7 & 4 & 5 & 20 & 25 \\
\hline $\begin{array}{c}\text { Operaciones } \\
\text { Unitarias }\end{array}$ & 6 & 6 & 6 & 8 & 6 & 5 & 6 & 8 & 25 & 26 \\
\hline Total & 17 & 18 & 17 & 18 & 14 & 17 & 13 & 16 & 60 & 69 \\
\hline Total por semestre & \multicolumn{2}{|c|}{35} & \multicolumn{2}{|c|}{35} & \multicolumn{2}{|c|}{30} & \multicolumn{2}{|c|}{29} & \multicolumn{2}{|c|}{130} \\
\hline
\end{tabular}

\section{Instrumentos de la investigación}

Las pruebas se realizaron en las instalaciones del grupo de investigación Nutrición, Salud y Calidad Alimentaria, de la Facultad de Ingeniería de la Universidad de Cartagena. A los alumnos se les suministró de manera presencial y en físico, una versión abreviada del cuestionario original ACRA realizado por Román y Gallego (2001). Este formato estuvo conformado por un total de 32 (EDA) agrupadas en cuatro grandes escalas (Adquisición, Codificación, Recuperación y Apoyo). Se realizó un análisis cuantitativo, asignándole a cada opción de respuesta un valor numérico: $A=1$ (Nunca o casi nunca); $B=2$ ( $A$ lgunas veces); $C=3$ (Bastantes veces); $D=4$ (Siempre o casi siempre). Este procedimiento fue similar a lo realizado por Bahamón et al., (2013).

\section{Análisis de datos}

Después de obtener la información sobre las EDA y las calificaciones de los estudiantes, se construyó una base de datos en el Programa Estadístico para Ciencias Sociales, IBM SPSS @ versión 19.0. Se analizaron los correspondientes promedios y desviaciones estándar. Se realizaron análisis de varianza ( $A N O V A$ unifactorial) suponiendo varianzas iguales entre las medias de las calificaciones y de las EDA por género; así mismo se empleó la prueba de Kruskal-Wallis para evaluar la hipótesis de que las medianas de los hombres y las mujeres dentro de cada una de las escalas de EDA eran iguales; manejando un nivel de significancia del $5 \%(\mathrm{p}<0,05)$. Y finalmente se realizó una correlación a partir de la prueba de $r$-Pearson, entre cada una de las escalas Adquisición, Codificación, Recuperación y Apoyo, con las calificaciones de 
los estudiantes, para estimar hasta qué nivel se relacionaron estas variables. La correlación se consideró altamente significativa al nivel de 0,01 (bilateral).

\section{RESULTADOS}

\section{Análisis de las calificaciones de la muestra de estudiantes que cursaron las asignaturas}

La Tabla 2, ilustra los promedios de las calificaciones finales de la muestra de estudiantes que cursaron las asignaturas. Se observan puntuaciones máximas de 4,33 - 4,53-4,79 en mujeres y de 4,30 - 4,56-4,57 para los hombres, en balance de materia, transporte de fluidos y operaciones unitarias respectivamente. Al tener en cuenta las calificaciones en la Universidad de Cartagena, se confirmó que dichas calificaciones estuvieron dentro del rango medio y alto. Ya que de 0,0 a 1,9 se clasificaron a los estudiantes en muy bajo; de 2,0 a 2,9 en bajo; de 3,0 a 3,7 en medio; 3,8 a 4,4 en medio/alto y de 4,5 a 5,0 en alto.

Tabla 2: Calificaciones promedios de la muestra de los estudiantes que cursaron las asignaturas

\begin{tabular}{|c|c|c|c|c|c|c|c|c|}
\hline Asignaturas & \multicolumn{2}{|c|}{ Balance de Materia } & \multicolumn{2}{|c|}{$\begin{array}{l}\text { Transporte de } \\
\text { Fluidos }\end{array}$} & \multicolumn{2}{|c|}{$\begin{array}{c}\text { Operaciones } \\
\text { Unitarias }\end{array}$} & \multicolumn{2}{|c|}{ Total } \\
\hline Género & $\begin{array}{l}\text { Hombre } \\
s(n=16)\end{array}$ & $\begin{array}{c}\text { Mujeres } \\
(n=18)\end{array}$ & $\begin{array}{l}\text { Hombre } \\
s(n=20)\end{array}$ & $\begin{array}{c}\text { Mujeres } \\
(n=25)\end{array}$ & $\begin{array}{l}\text { Hombre } \\
s(n=25)\end{array}$ & $\begin{array}{c}\text { Mujeres } \\
(n=26)\end{array}$ & $\begin{array}{l}\text { Hombre } \\
\mathrm{s}(\mathrm{n}=60) \\
\end{array}$ & $\begin{array}{c}\text { Mujeres } \\
(n=69) \\
\end{array}$ \\
\hline Promedio & 3,59 & 3,56 & 3,67 & 3,62 & 3,74 & 3,86 & 3,67 & 3,68 \\
\hline Desviación estándar & 0,71 & 0,77 & 0,89 & 0,91 & 0,83 & 0,93 & 0,81 & 0,87 \\
\hline $\begin{array}{l}\text { (\%) Coeficiente } \\
\text { Variación }\end{array}$ & 19,78 & 21,63 & 24,25 & 25,14 & 22,19 & 24,09 & 22,09 & 23,64 \\
\hline Mínimo & 2,88 & 2,79 & 2,78 & 2,71 & 2,91 & 2,93 & 2,86 & 2,81 \\
\hline Máximo & 4,30 & 4,33 & 4,56 & 4,53 & 4,57 & 4,79 & 4,48 & 4,55 \\
\hline Estadísticos & \multicolumn{2}{|c|}{$\begin{array}{c}t=3,89 \\
(p=0,38>0,05)\end{array}$} & \multicolumn{2}{|c|}{$\begin{array}{c}t=3,05 \\
(p=0,47>0,05)\end{array}$} & \multicolumn{2}{|c|}{$\begin{array}{c}t=3,45 \\
(p=0,52>0,05)\end{array}$} & \multicolumn{2}{|c|}{$\begin{array}{c}t=3,99 \\
(p=0,82>0,05)\end{array}$} \\
\hline
\end{tabular}

Al comprar los promedios de las calificaciones en cada asignaturas y de manera global, se comprobó que no hubo diferencias estadísticamente significativas entre hombres y mujeres $(p>0,05)$. En las mujeres las calificaciones estuvieron entre 2,71 y 4,79 y de 2,78 y 4,57 en los hombres. Lo que indica que hubo estudiantes que no aprobaron los contenidos temáticos de las respectivas asignaturas; así como otros que se adaptaron a la metodología de enseñanza del docente y obtuvieron mejores calificaciones.

\section{Análisis de las EDA entre hombres y mujeres}

En la Tabla 3, Se esquematizan los ANOVA, realizados entre los valores promedios de los hombres y las mujeres en cada escala de EDA. El análisis indicó mayor dispersión en los datos obtenidos en las mujeres, ya que los estadísticos suma de cuadrados y cuadrados medios entre grupos e intra grupos fueron más altos.

Tabla 3. Análisis de varianza unifactorial entre las EDA por géneros

\begin{tabular}{|l|c|c|c|c|c|}
\hline \multicolumn{7}{|c|}{ ANOVA para Hombres por Escalas - EDA } \\
\hline \multicolumn{1}{|c|}{ Fuente } & Suma de Cuadrados & GL & Cuadrado Medio & Razón- $F$ & Valor- $P$ \\
\hline Entre grupos & 1095,09 & 3 & 365,031 & 18,49 & 0,0006 \\
\hline Intra grupos & 157,935 & 8 & 19,7418 & & \\
\hline Total & 1253,03 & 11 & & & \\
\hline \multicolumn{7}{|c|}{ ANOVA para Mujeres por Escalas - EDA } \\
\hline \multicolumn{7}{|c|}{ Fuente } & Suma de Cuadrados & GL & Cuadrado Medio & Razón- $F$ & Valor- $P$ \\
\hline Entre grupos & 3521,72 & 3 & 1173,91 & 17,27 & 0,0007 \\
\hline Intra grupos & 543,635 & 8 & 67,9543 & & \\
\hline Total & 4065,35 & 11 & & & \\
\hline
\end{tabular}

Se observó que la razón-F, para los hombres fue de 18,49 y para las mujeres igual a 17,27. Por lo que en ambos casos el valor-p fue menor que 0,05 estableciéndose que si hubo diferencias estadísticamente significativa entre las medias de ambos géneros en las respectivas escalas de EDA. Así mismo con la Prueba de Kruskal-Wallis se compararon las medianas en lugar de las medias dentro de cada escala EDA, y se encontraron diferencias estadísticamente significativas tanto para hombres (Estadístico $=10,01$ y $\mathrm{p}=0,02<0,05$ ) como para mujeres (Estadístico $=9,15$ y $\mathrm{p}=0,03<0,05$ ). Indicando que el género si influye en las elección de las EDA de los estudiante. La prueba de comparación múltiple indicó entre cuales medias hubo diferencias estadísticas. 
En la Tabla 4, se pueden observar los resultados por género obtenidos con el cuestionario ACRA. La preferencia general estuvo orientada hacia la escala de codificación y apoyo, tanto para los hombres como para las mujeres, en todas las asignaturas. Los datos indican la media ( \pm ) con su respectiva desviación estándar. *Letras distintas en una misma fila indican diferencias estadísticamente significativas $(p<0,05)$, según test HSD de Tukey al $5 \%$.

Tabla 4: Estrategias de aprendizaje de los estudiantes por asignatura y género

\begin{tabular}{|c|c|c|c|c|c|c|c|c|}
\hline \multirow{2}{*}{$\begin{array}{c}\text { Estrategias } \\
\text { de } \\
\text { aprendizaje }\end{array}$} & \multicolumn{2}{|c|}{ Balance de Materia } & \multicolumn{2}{|c|}{ Transporte de Fluidos } & \multicolumn{2}{|c|}{ Operaciones Unitarias } & \multicolumn{2}{|c|}{ Totales } \\
\hline & $\begin{array}{c}\text { Hombres } \\
(n=16)\end{array}$ & $\begin{array}{c}\text { Mujeres } \\
(n=18)\end{array}$ & $\begin{array}{c}\text { Hombres } \\
(n=20)\end{array}$ & $\begin{array}{c}\text { Mujeres } \\
(n=25)\end{array}$ & $\begin{array}{c}\text { Hombres } \\
(n=25)\end{array}$ & $\begin{array}{c}\text { Mujeres } \\
(n=26)\end{array}$ & $\begin{array}{c}\text { Hombres } \\
(n=60)\end{array}$ & $\begin{array}{c}\text { Mujeres } \\
(\mathrm{n}=69)\end{array}$ \\
\hline Adquisición & $\begin{array}{c}50,67 \pm 2,8 \\
9 a\end{array}$ & $\begin{array}{c}52,67 \pm 2 \\
35 a\end{array}$ & $\begin{array}{c}56,62 \pm 3,1 \\
9 \mathrm{a}\end{array}$ & $\begin{array}{c}63,33 \pm 3,5 \\
1 \mathrm{~b}\end{array}$ & $\begin{array}{c}49,67 \pm 4,7 \\
2^{\underline{a}}\end{array}$ & $\begin{array}{c}65,41 \pm 2,6 \\
5 b\end{array}$ & $\begin{array}{c}52,32 \pm 3,7 \\
6 a \\
\end{array}$ & $\begin{array}{c}60,47 \pm 6 \\
83 \mathrm{~b} \\
\end{array}$ \\
\hline Codific & $\begin{array}{c}77,33 \pm 4,6 \\
3 a\end{array}$ & $\begin{array}{c}98,33 \pm 4 \\
04 b\end{array}$ & $\begin{array}{c}75,36 \pm 2,6 \\
9 \underline{a}\end{array}$ & $\begin{array}{c}88,65 \pm 3,7 \\
9 c\end{array}$ & $\begin{array}{c}63,67 \pm 1,2 \\
4 d\end{array}$ & $\begin{array}{c}96,03 \pm 5,9 \\
6 b\end{array}$ & $\begin{array}{c}72,12 \pm 7,8 \\
3 a\end{array}$ & $\begin{array}{c}94,34 \pm 5 \\
05 \mathrm{~b}\end{array}$ \\
\hline $\begin{array}{c}\text { Recuperaci } \\
\text { ón }\end{array}$ & $\begin{array}{r}45,42 \\
\pm 3,20 \mathrm{a} \\
\end{array}$ & $\begin{array}{c}49,72 \pm 2 \\
35 a \\
\end{array}$ & $\begin{array}{c}48,33 \pm 2,3 \\
5^{\mathbf{a}}\end{array}$ & $\begin{array}{c}53,64 \pm 3,7 \\
9 a b\end{array}$ & $\begin{array}{c}47,05 \pm 1,3 \\
5^{\mathbf{a}}\end{array}$ & $\begin{array}{c}39,83 \pm 3,7 \\
9 c\end{array}$ & $\begin{array}{c}46,93 \pm 1,4 \\
6 a\end{array}$ & $\begin{array}{c}47,73 \pm 7 \\
12 a\end{array}$ \\
\hline Apoyo & $\begin{array}{c}57,67 \pm 2,2 \\
3 b\end{array}$ & $\begin{array}{c}64,6364 \\
63 a\end{array}$ & $\begin{array}{c}59,67 \pm 2,2 \\
3 b\end{array}$ & $\begin{array}{c}79,36 \pm 4,5 \\
7 c\end{array}$ & $\begin{array}{c}59,67 \pm 3,5 \\
4 \mathrm{~b}\end{array}$ & $\begin{array}{c}78,21 \pm 2,5 \\
7 c\end{array}$ & $\begin{array}{c}61,32 \pm 2,8 \\
6 a\end{array}$ & \begin{tabular}{|c|}
$71,71 \pm 1$ \\
$2,21 \mathrm{~b}$
\end{tabular} \\
\hline
\end{tabular}

En las Figura 1, y Figura 2, se observan los diagramas de cajas, que comparan las medias obtenidas entre los hombres y las mujeres en las escalas de EDA. Se observó que en los hombres en hubo mayor dispersión en los valores de las escala de codificación, presentando las mayores puntuaciones. Mientras que en las mujeres hubo mayor dispersión en los valores de la escala de apoyo; y los promedios más altos estuvieron también en la escala de codificación.

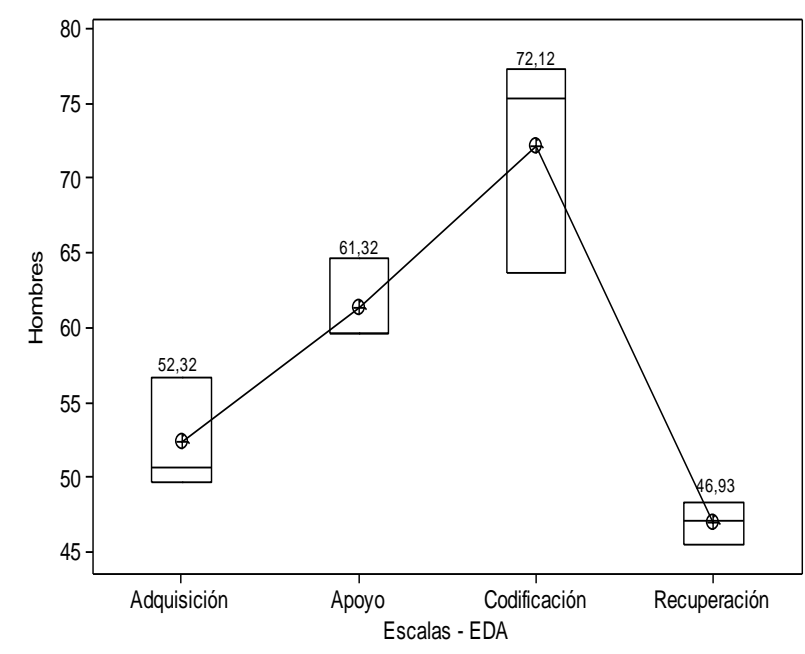

Fig. 1: Valores promedios en las escalas EDA en los Hombres

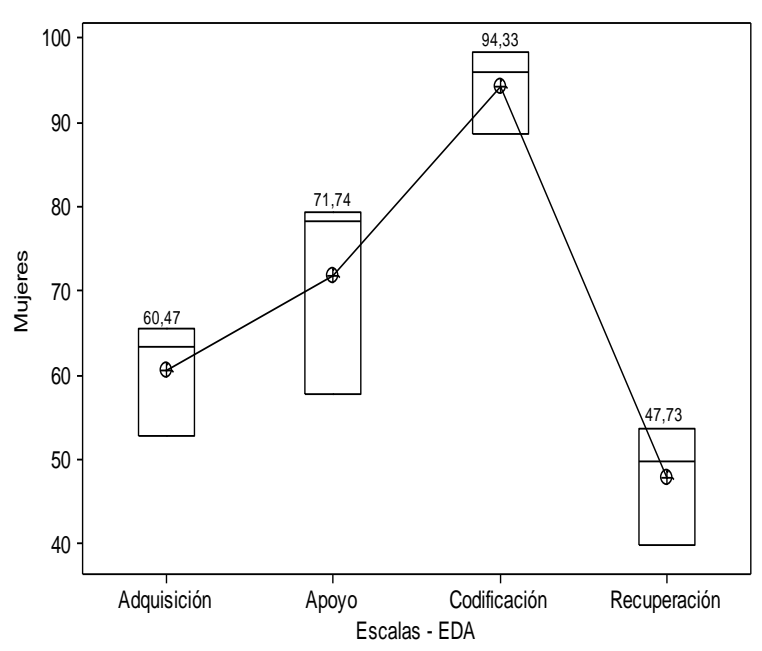

Fig. 2: Valores promedios en las escalas EDA en las Mujeres

Al realizar un análisis más detallado por escala, se pudo notar que los estudiantes evaluados tanto hombres como mujeres en las tres asignaturas de balance de materia, transporte de fluidos y operaciones unitarias, indicaron en la escala de adquisición, que las estrategias más utilizadas fueron: el subrayado lineal, seguido por la exploración teórica, repaso mental, y en voz alta. Lo cual es importante para el desarrollo cognitivo de los estudiantes, y concuerda con lo encontrado y afirmado por Pestana y Martínez, (2013) quienes aseguraron que estas EDA son indispensables en estas áreas del saber y ayudan a potenciar la memoria a corto plazo.

En la escala de codificación, las EDA más utilizadas por los estudiantes en ambos géneros fueron: nemotecnias, elaboración imágenes, autopreguntas y parafraseado. Así como organización, agrupamientos, mapas conceptuales y elaboración de diagramas de flujo; estrategias que son de vital importancia para que los estudiantes puedan manejar gran cantidad de información en sus respectivas áreas del saber, sobre todo en ingeniería (Muelas y Navarro, 2015). Dentro de la escala de recuperación destacaron las EDA de planificación de respuestas; mientras que en las EDA de apoyo más utilizadas fueron: automanejo, autoconocimiento, autoinstrucción y autocontrol, también se observaron preferencias por la motivación intrínseca y extrínseca. 
Bahamón et al., (2013) señala que las estrategias de recuperación y apoyo se ubican en las más importantes para el desarrollo mental de los estudiantes; así mismo son las que permiten relacionar lo aprendido con lo ya conocido y posibilitan la reflexión pausada y consciente de lo que se va aprendiendo. Es importante destacar que las mujeres en cada una de las escalas analizadas, presentaron valores más altos que los hombres, lo cual indica mayor capacidad de estas, al momento de procesar la información, especialmente con las estrategias de codificación, lo que podría ser atribuido a que estas suelen ser más organizadas que los hombres en sus hábitos de estudio y aprendizaje (Gallo et al., 2014). En general las tres asignaturas implicaron una alta exigencia académica y requieren manejar gran cantidad de información en un lenguaje ingenieril, con fórmulas matemáticas y diagramas de flujo de procesos. Por lo tanto es indispensable que los estudiantes desarrollen capacidades de abstracción y meta-cognición, utilizando EDA como las de codificación, que según Gallo et al., (2014) ayudan en la organización, clasificación, entendimiento y logro de los objetivos académicos. Y las de apoyo que según lo encontrado por Ossa y Aedo, (2014) constituyen una fase superior porque requieren de un pensamiento complejo, de un análisis más significativo y de mayor abstracción mental.

Los resultados del presente trabajo con estudiantes de ingeniería de alimentos, fueron similares a los reportados por Bahamón et al., (2013) quienes aplicaron el cuestionario ACRA, a estudiantes universitarios de Boyacá (Colombia) y encontraron un $42,6 \%$ de preferencia por la escala de codificación, seguida por la de apoyo con un 16,3\%. Así mismo Medina et al., (2014) señalaron que las estrategias más utilizadas por los estudiantes de Ingeniería agrónomica fueron: las de codificación y apoyo, con el uso del agrupamiento, la aplicación de la información, el automanejo/regulación y evaluación. En esta misma línea investigativa López-Aguado, (2011) y Gargallo et al., (2012) encontraron que estudiantes universitarios utilizaron en mayor medida las estrategias de codificación y apoyo, con técnicas como elaboración de mapas conceptuales, auto-preguntas y agrupamientos de la información, así como auto-instrucciones, contradistractores y automanejo.

Por su parte Quispe et al., (2014) encontraron que el 41,7\% de los estudiantes utilizaron estrategias de apoyo, el $36 \%$ de recuperación, $14,4 \%$ de adquisición y 7,9\% de codificación. Indicaron que los estudiantes que tuvieron promedio normal, elaboraron resúmenes y esquemas como principal estrategia de aprendizaje, mientras que los de mejores calificaciones poseían más estrategias de apoyo y codificación. Por su parte Loret de Mola (2011) en estudiantes universitarios, detectó preferencias en las estrategias de codificación en $49 \%$, seguidas por las de adquisición con 48\%, las de recuperación con $47 \%$ y las de apoyo con $37 \%$. Pestana y Martínez (2013) identificaron las preferencias de EDA en los estudiantes universitarios de Sucre (Colombia) y hallaron que la escala de adquisición fue la de mayor prevalencia, con un porcentaje que osciló entre $30-43 \%$, seguida de la escala de apoyo con un porcentaje entre $28-32 \%$.
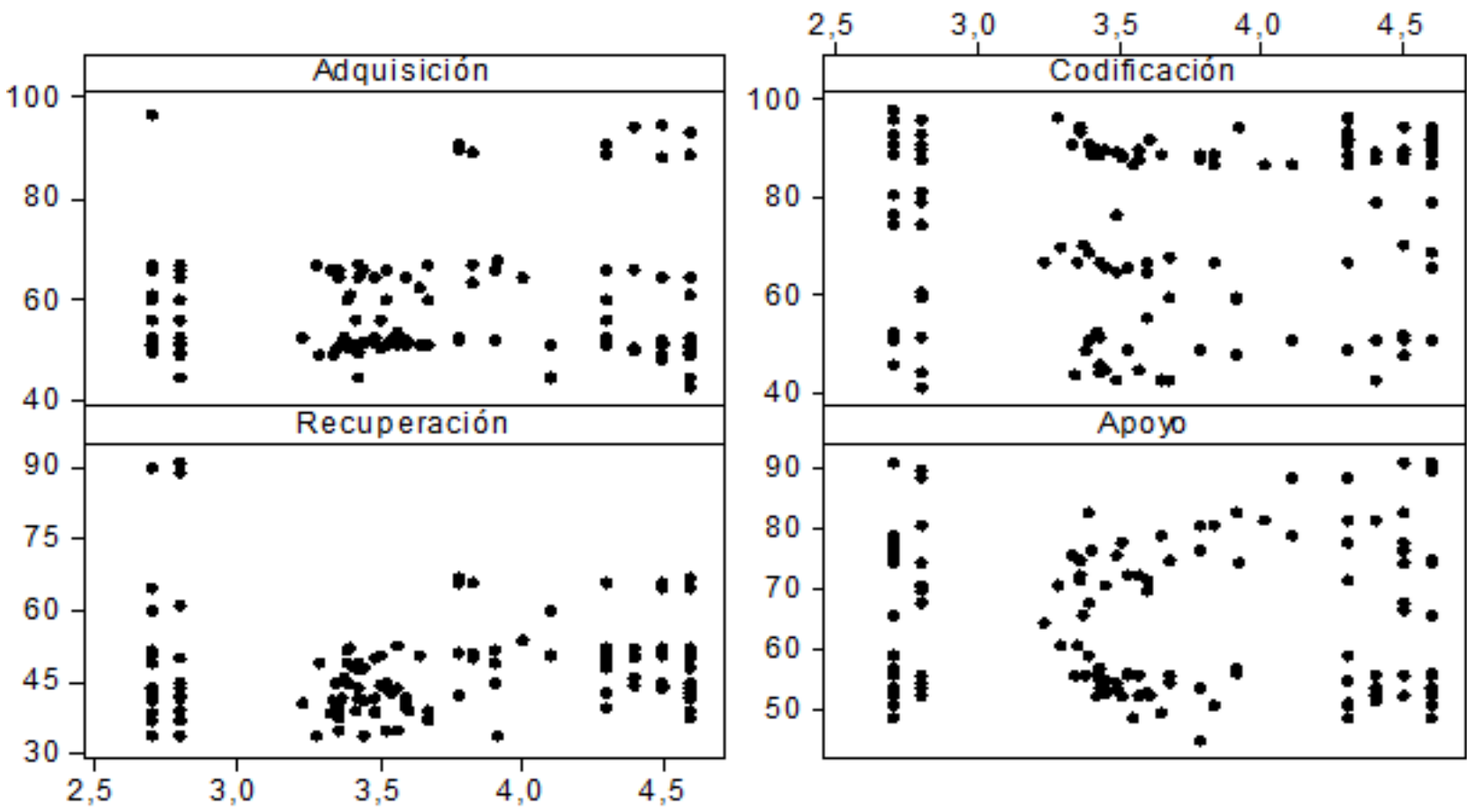

Fig. 3: Relación entre EDA con las calificaciones de los estudiantes 


\section{Relación entre las calificaciones de los estudiantes y las EDA}

La Figuras 3, se muestra la relación entre las escalas de aprendizajes con las calificaciones de los estudiantes. La nube de puntos para cada escala se encontró dispersa en el plano, con una tendencia levemente positiva (casi nula). Para analizar en detalle tal relación se usó la prueba de Pearson a una significancia del $1 \%(p \leq 0,01)$. Con las respectivas hipótesis de $\mathrm{H}_{0}$ : $r$-Pearson $=0$ (la referida escala de estrategia de aprendizaje y las calificaciones no se encuentran relacionados linealmente). $\mathrm{Y}_{\mathrm{H}}$ : $\mathrm{r}$-Pearson $\neq$ 0 (la escala de estrategia de aprendizaje y las calificaciones se encuentran relacionados linealmente).

En la Tabla 5, se enmarcan las correlaciones entre cada escala de EDA con las calificaciones por géneros. En la parte superior de la Tabla 5 , se encuentran los datos de los hombres, en los cuales se puede ver que ninguna de las escalas presentó una correlación significativa con las calificaciones $(p>0,01)$ siendo directa pero no significativa en la de codificación $(r=0,45$ y $p=0,13)$ y totalmente nula en la de recuperación $(r=0,00$ y $p=0,99$ ). Los hombres que más utilizaron la escala de apoyo, así mismo lo hicieron con la de codificación, cuya relación fue directa y altamente significativa $(r=0,89$ y $p=0,00)$. Resultados que fueron similares a los encontrados por Bahamón et al., (2013), Medina et al., (2014), Quispe et al., (2014), Pestana y Martínez, (2013) y Case et al., (2010).

Tabla 5: Correlación entre calificaciones y las EDA por género, $\left({ }^{*} \mathrm{p}<0,05 ;{ }^{* *} \mathrm{p}<0,01\right)$

\begin{tabular}{|c|c|c|c|c|c|c|}
\hline \multicolumn{2}{|c|}{$\begin{array}{l}\text { Hombres }(n=60) \\
\text { Mujeres }(n=69)\end{array}$} & $\begin{array}{l}\text { Calificaciones } \\
\text { estudiantes }\end{array}$ & Adquisición & Codificación & Recuperación & Apoyo \\
\hline \multirow{2}{*}{$\begin{array}{c}\text { Calificaciones } \\
\text { estudiantes }\end{array}$} & r-Pearson & 1 & 0,14 & 0,45 & 0,00 & $-0,17$ \\
\hline & p-valor & & 0,57 & 0,13 & 0,99 & 0,49 \\
\hline \multirow{2}{*}{ Adquisición } & r-Pearson & 0,04 & 1 & 0,03 & 0,10 & 0,07 \\
\hline & p-valor & 0,89 & & 0,91 & 0,71 & 0,79 \\
\hline \multirow{2}{*}{ Codificación } & r-Pearson & 0,39 & $0,59^{* *}$ & 1 & 0,22 & $0,89^{\star *}$ \\
\hline & p-valor & 0,12 & 0,01 & & 0,38 & 0,00 \\
\hline \multirow{2}{*}{ Recuperación } & r-Pearson & 0,21 & $-0,28$ & $0,73^{\star *}$ & 1 & 0,00 \\
\hline & p-valor & 0,23 & 0,27 & 0,00 & & 0,99 \\
\hline \multirow{2}{*}{ Apoyo } & r-Pearson & 0,01 & $0,86^{* *}$ & 0,25 & 0,24 & 1 \\
\hline & $\mathrm{p}$-valor & 0,70 & 0,00 & 0,32 & 0,35 & \\
\hline
\end{tabular}

Al analizar los datos de las mujeres, esquematizados en la parte inferior de la Tabla 5 , se observó que las escalas de adquisición, recuperación, y apoyo no se correlacionaron con las calificaciones de las estudiantes $(p>0,01)$. En la escala de codificación se encontró una relación directa, pero no significativa $(r=0,39$ y $p=0,12)$ lo cual indica que las mujeres de tienden a usar más las estrategias de codificación. Al observar los datos entre las escalas, se observó que las mujeres utilizaron las estrategias de apoyo, emplearon proporcionalmente las de adquisición $(r=0,86$ y $p=0,00)$. Así mismo se observó correlación entre las escalas de EDA de codificación con las de adquisición $(r=0,59$ y $p=0,00)$. Estos resultados fueron similares a los reportados por Loret de Mola (2011), en su trabajo con estudiantes en una universidad peruana. Así mismo con los hallados por Muelas y Navarro (2015) quienes encontraron correlación entre las EDA del cuestionario ACRA y las calificaciones de estudiantes universitarios.

\section{CONCLUSIONES}

Las mujeres tuvieron mejores calificaciones en las asignaturas de balance de materia y operaciones unitarias; mientras que los hombres destacaron en transporte de fluidos. Al comprar los promedios de las calificaciones en cada asignaturas se comprobó que no hubo diferencias estadísticamente significativas entre hombres y mujeres $(p>0,05)$. La preferencia general en las estrategias de aprendizajes (EDA) estuvo orientada hacia las escalas de codificación y apoyo; siendo las mujeres las que presentaron promedios más altos en dichas escalas, en las tres asignaturas analizadas. Entre hombres y mujeres hubo diferencias estadísticamente significativas en cada una de las escalas de adquisición, codificación, recuperación y apoyo $(p<0,05)$, por lo que se establece que el género si influye en la elección de las respectivas EDA. No hubo correlación signifcativa entre las calificaciones de los estudiantes y cada una de las escalas de EDA analizadas $(p>0,05)$. Identificar las EDA es una alternativa para mejorar las metodologías de enseñanzaaprendizaje en las asignaturas de balance de materia, transporte de fluidos y operaciones unitarias.

\section{REFERENCIAS}

Alkhateeb, H. y R. Nasser, Assessment of learning and study strategies of university students in Qatar using an Arabic translation of the learning and study strategies inventory, Psychological Reports, Sociocultural Issues in Psychology, 114(3), 947-965 (2014) 
Bahamón, M., M. Vianchá., L. Alarcón y C. Bohórquez, Estilos y estrategias de aprendizaje, relacionadas con el logro académico en estudiantes universitarios, Pensamiento Psicológico, 11(1), 115-129 (2013)

Casé, R., H. Neer., S. Lopetegui y S. Doná, Estrategias de aprendizaje y rendimiento académico según el género en estudiantes universitarios, Revista de Psicología, 11(1), 199-211 (2010)

Gallo, B. y otros cinco autores, Metodología centrada en el aprendizaje. Su impacto en las estrategias de aprendizaje y en el rendimiento académico de los estudiantes universitarios, Revista Española de Pedagogía, 72(259), 415-435 (2014)

Gargallo, B. y otros tres autores, Estrategias de aprendizaje en estudiantes Universitarios excelentes y medios. Su evolución a lo largo del primer año de carrera, Revista Electrónica de Investigación y Evaluación Educativa, 18(2), 1-21 (2012)

López-Aguado, M., Estrategias de aprendizaje en estudiantes universitarios. Diferencias por género, curso y tipo de titulación, Teoría de la educación y en la sociedad del conocimiento, 12 (2), 203-233 (2011)

López, P.L., Población muestra y muestreo, Punto Cero, 69-74 (2004)

Loret de Mola, J.E., Estilos y Estrategias de Aprendizaje en el Rendimiento Académico de los estudiantes de la Universidad peruana "Los Andes" (Perú), Revista Estilos de Aprendizaje, 8 (8), 149-184 (2011)

Marugán, M. y otros tres autores, Estrategias cognitivas de elaboración y naturaleza de los contenidos en estudiantes universitarios, Psicología Educativa, 19(1), 13-20 (2013)

Medina, A. y otros tres autores, Evaluación de las estrategias de aprendizaje en la Licenciatura de Ingeniero Agrónomo, Revista Iberoamericana de Ciencias, 1 (5), 165-173 (2014)

Muelas, A. y E. Navarro, Learning Strategies and Academic Achievement, Procedia-Social and Behavioral Sciences, 165 (6). 217-221 (2015)

Ossa, C. y S. Aedo, Enfoques de aprendizaje, autodeterminación y estrategias metacognitivas en estudiantes de pedagogía de una Universidad Chilena, Ciencias Psicológicas, 8(1), 79-88 (2014)

Pestana, P. y J. Martínez, Preferencia de estilos y uso de estrategias de aprendizaje en los estudiantes de la Universidad de Sucre, REVISALUD, 1(1) 45-57 (2013)

Pool-Cibrian, J. y J. Martínez-Guerrero, Autoeficacia y uso de estrategias para el aprendizaje autorregulado en estudiantes universitarios, Revista Electrónica de Investigación Educativa, 15(3), 21-37 (2013)

Quispe, Z., Pérez, D. y Y. Chinarro, Estrategias de aprendizaje de los estudiantes de la facultad de enfermería de la Universidad Nacional San Luis Gonzaga de Ica, y su relación con el rendimiento académico, ciclo 2012-II, Revista enfermería a la Vanguardia, 2(1), 33-38 (2014)

Roman, J. M. y Gallego, S., Escalas de Estrategias de Aprendizaje. Manual ACRA. Publicaciones de Psicología Aplicada. Madrid: TEA Ediciones (2001)

Villardón-Gallego, L. y tres autores, Learning Competence in University: Development and Structural Validation of a Scale to Measure, Revista de Psicodidáctica, 18(2), 357-374 (2013)

Quispe, Z., Pérez, D. y Y. Chinarro, Estrategias de aprendizaje de los estudiantes de la facultad de enfermería de la Universidad Nacional San Luis Gonzaga de Ica, y su relación con el rendimiento académico, ciclo 2012-II, Revista enfermería a la Vanguardia, 2(1), 33-38 (2014)

Villardón-Gallego, L. y tres autores, Learning Competence in University: Development and Structural Validation of a Scale to Measure, Revista de Psicodidáctica, 18(2), 357-374 (2013) 